\title{
9 Quality assurance in the Trusted Third Party
}

The declaration of consent is considered a central legal document in order to legitimise the processing of study participant data. Therefore, quality assurance measures are carried out on these documents and data in the TTP. As mentioned above, the TTP sends monthly quality reports with specific deficiencies on declarations of consent that require revision. These can be misspelled names, missing signatures or insufficiently marked consents.

The Trusted Third Party of the DZHK currently exclusively supervises multicentre studies (more than 120 affiliated centres). The reports are generated on the basis of the study and the centre and are currently transmitted via encrypted communication to a contact person of the student assistant and must be distributed from there.

Is the above procedure sufficient to ensure a centrally controlled verification of the consent status for the release of data and samples? What further measures might need to be taken?

The procedure described is considered admissible. The GDPR does not provide any explicit stipulations for the consent management. However, it is crucial for the execution of the research project that errors are avoided and data integrity is ensured. The processing of the personal data in the sense of an examination, return transmission, correction and renewed examination as well as further processing is therefore a procedure necessary for reaching the pur- 
Part II of the legal opinions: Detailed Questions on organisational and technical measures

pose of the research project. This must be considered as covered by the declaration of consent.

Encrypted transmission of declarations of consent is required, since the declarations of consent themselves may already qualify as health data. In addition to transport encryption, the data itself should be encrypted so that it cannot be read in the event of intermediate storage by a telecommunications service provider. Keys for decryption should be transmitted via an alternative means of communication. 